\title{
Paraplegia espástica causada por cisticercose intramedular: apresentação de caso e breve revisão bibliográfica
}

\author{
Luciano Ferreira de Holanda', Benedito Jamilson Araújo Pereira², Rafael \\ Rodrigues Holanda ${ }^{3}$, Amauri Pereira da Silva Filho ${ }^{3}$, José da Costa Leão \\ Segundo ${ }^{3}$, Pierre Vansant Oliveira Eugênio ${ }^{4}$, Gabriela Nascimento Holanda ${ }^{5}$ \\ Serviço de Neurocirurgia do Hospital Antônio Targino, Campina Grande, PB, Brasil.
}

\section{RESUMO}

Relato de cisticercose intramedular em homem de 42 anos que há seis anos sentia parestesias em ambas as pernas, que evoluíram para ausência de sensação dolorosa, por fim paraparesia espástica dos membros inferiores. Ao exame físico, verificaram-se sinal de Babinski bilateral, espasticidade dos membros inferiores e déficit de sensibilidade abaixo de T7. Ressonância nuclear magnética mostrou uma lesão expansiva ao nível de T5. Durante a exérese do processo, constatou-se que se tratava de cisticercose, em sua forma tumoral intramedular. Após a retirada, o mesmo evoluiu de maneira satisfatória, porém sem regressões dos sintomas.

\section{PALAVRA-CHAVE}

Neurocisticercose intramedular.

\section{ABSTRACT}

Spastic paraplegia due to spinal cord cisticercosis: case report

A case of spinal cord cysticercosis in a 42 year-old man is reported. He presented a six years history of paresthesia in both legs, which progressed to lack of feeling pain, spastic paraparesis of the lower limbs. On physical examination there was bilateral Babinski sign, spasticity of the lower limbs, and lack of sensitivity below T7. The magnetic resonance imaging showed an expansive lesion at the level of T5. Patient was referred for excision of the process, and while it was withdrawn, it was found that to be a cysticercosis in a shape of spinal cord tumor. There was no postoperative complication but the symptoms did not improve.

\section{KEYWORD}

Spinal cord neurocysticercosis.

\section{Relato do caso}

Paciente SC, 42 anos, sexo masculino, natural e procedente de Picuí, PB, procurou atendimento médico no dia 27/1/2009, com queixa de não mais conseguir andar, além de sentir que as pernas apresentavam-se rígidas; referiu que há seis anos começou a sentir parestesias em ambas as pernas, que evoluíram para paraparesia crural, com marcha atáxica, no último ano, com piora acentuada no mês de dezembro de 2008, quando se encontrara paraplégico e com perda do controle vesical, ora apresentando plenitude vesical, ora perdendo o controle miccional. Ele ainda relatou que há mais ou menos três anos chegava a se exibir perante amigos, arrancando pelos e ficando próximo a objetos quentes sem sentir dor.

Ao exame físico, observou-se déficit sensitivo e motor abaixo do dermátomo $\mathrm{T} 7$, com paraplegia espástica dos membros inferiores, sinal de Babinski bilateral e perda do controle vesical. A ressonância nuclear mag-

Doutorando em Medicina pela Universidade Federal da Bahia (UFBA).

2 Residente em Neurocirurgia - equipe CENNA (Centro Especializado em Neurologia e Neurocirurgia, Hospital Beneficência Portuguesa de São Paulo, SP).

3 Neurocirurgião do Hospital Antônio Targino.

4 Acadêmico de Medicina da Universidade Federal de Campina Grande (UFCG).

5 Otorrinolaringologista do Hospital Antônio Targino. 
nética (RNM) mostrou discreta acentuação da cifose dorsal fisiológica, evidência de lesão intradural com características predominantemente císticas, localizadas entre T5-T6, promovendo certa expansão do parênquima no local com realce pelo gadolínio, cabendo, no diagnóstico diferencial, tumoração de linhagem astroglial e processo inflamatório atípico (Figura 1). O paciente foi encaminhado para cirurgia no dia 16/2/2009. A equipe realizou laminectomia entre T4-T7 para retirada do processo expansivo e, quando da incisão da dura-máter, o processo expansivo se apresentou e, pelas características, viu-se que se tratava de cisticerco (Figura 2), diagnóstico confirmado posteriormente pelo exame anatomopatológico. Após o procedimento, o paciente evoluiu de maneira satisfatória, recebendo alta hospitalar em 19/2/2009, com a prescrição de albendazol $400 \mathrm{mg}$ de $8 / 8$ horas, durante 21 dias, por conta da baixa penetração desse fármaco na medula espinhal, associado com dexametasona, também por 21 dias. Apesar do sucesso da cirurgia, não houve regressão dos sintomas previamente apresentados.
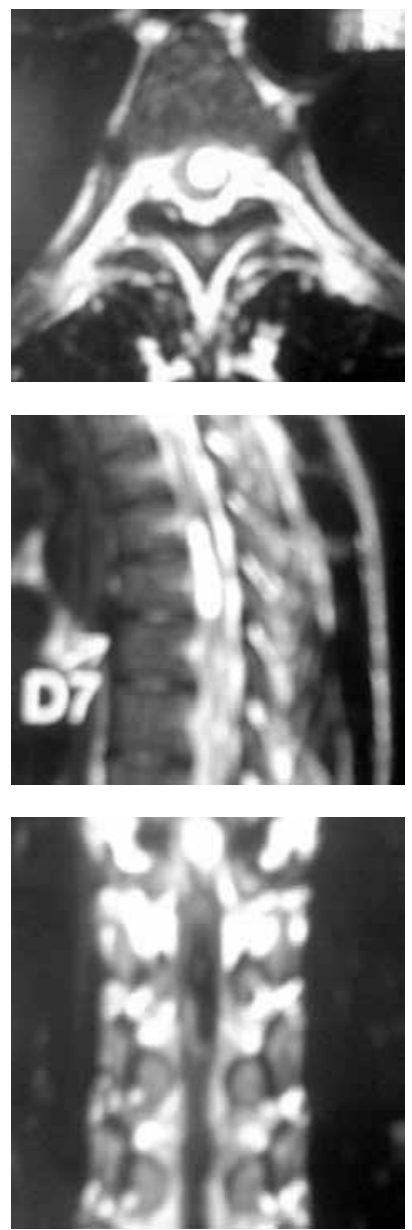

Figura 1-RNM da coluna torácica evidenciando lesão intradural.

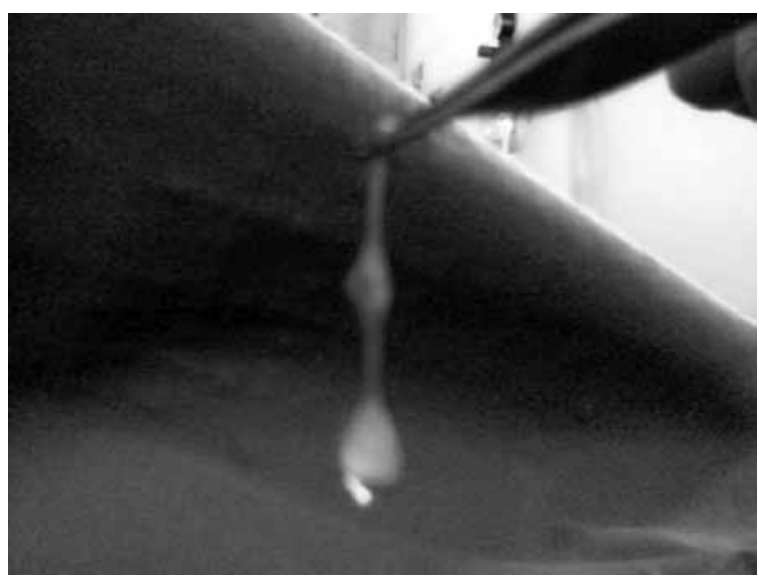

Figura 2 - Cisticerco retirado após laminectomia.

\section{Discussão}

A cisticercose é um sério problema de saúde pública, decorrente da infecção do homem pela larva Cysticercus cellulosae, do parasita Taenia solium e muito raramente da Taenia saginata. É a mais grave parasitose do sistema nervoso central, por causa da sua alta incidência e limitação terapêutica. ${ }^{14}$ Sobrevém em qualquer faixa etária, sem distinção de raça, sexo, cor ou classe socioeconômica. É endêmica em várias partes do planeta, particularmente na América Latina, África e Ásia, e ainda relativamente habitual em Portugal, Espanha e países do Leste Europeu. ${ }^{18,23}$ Os fatores epidemiológi$\cos$, tais como criação de porcos, manejo e consumo de carne suína infectada, mau acondicionamento de dejetos humanos, proximidade de chiqueiros a hortas, jardins ou fontes de água, deglutição de verduras ou frutas mal lavadas, são importantes elementos da anamnese. A contaminação faz-se por auto ou heteroinfestação. ${ }^{8}$

Durante o ciclo biológico da Taenia solium, o homem pode virar hospedeiro casual de sua configuração larvária, seja pelo consumo ou por autoinfestação interna; os ovos produzidos pelo verme adulto impetram o estômago, onde se irrompem pela ação do suco gástrico. Os embriões são liberados e, penetrando na parede do intestino delgado, alcançam a circulação. $\mathrm{O}$ embrião vai se instalar preferencialmente no tecido neural, no tecido subcutâneo, no músculo esquelético e no globo ocular, onde cresce na forma larvária, o Cysticercus cellulosae. ${ }^{20} \mathrm{~A}$ cisticercose é a nosologia parasitária que mais acomete o sistema nervoso central (SNC). ${ }^{10} \mathrm{O}$ cometimento espinhal ocorre em $0,7 \%$ a $5,8 \%$ dos pacientes, e a forma intramedular é uma rara manifestação. ${ }^{3,7,13,17,20}$ Apesar de corriqueiramente estar anexa às lesões cerebrais, pode apresentar-se isoladamente. ${ }^{7}$ Pode ser classificada em: vertebral, extradural, leptomeníngea (subaracnóidea) e intramedular, ${ }^{13}$ sendo a extradural de ocorrência excepcional e as formas 
leptomeníngeas, seis a oito vezes mais comuns que as intramedulares. ${ }^{20}$

Até o ano de 1998, foram relatados na literatura mundial 45 casos de cisticercose intramedular. ${ }^{7}$ Parece existir uma proporcionalidade entre o fluxo sanguíneo referente a cada região da medula e a ocorrência de cisticercose intramedular. ${ }^{7,15,20}$ Assim, prepondera a localização torácica $(55,1 \%)$, acompanhada pela cervical $(24,1 \%)$ e, finalmente, pela lombossacra $(20,6 \%),{ }^{20}$ que corresponde exatamente ao nosso caso. A migração da larva inferiormente ao cérebro, para o espaço subaracnóideo ${ }^{13}$, e a via hematogênica venosa ${ }^{17}$ são conjecturas que tentam elucidar a forma leptomeníngea. A dispersão hematogênica e a via ventrículo-ependimária têm sido postuladas como meios de a larva atingir o interior da medula. ${ }^{7,13,17,20}$ A teoria hematogênica é a mais sedutora, pois, sendo os cisticercos distribuídos no hospedeiro pela corrente circulatória, esta levaria os parasitas ao tecido medular. ${ }^{20}$ As dessemelhantes incidências entre o cérebro e a medula tornam manifesta a apresentação de outros fatores. Dentre esses, o fluxo sanguíneo cerebral é cerca de cem vezes superior ao medular, ${ }^{15}$ os vasos da medula têm calibre delgado e baixa pressão, o que não beneficia fenômenos embólicos, e a diminuição de expansibilidade no tecido nervoso da medula, quando comparado ao cérebro, dificulta a presença desse cisto, nesta localização. ${ }^{20}$

As manifestações clínicas da cisticercose raquiana mais frequentes são sinais e sintomas de constrição da medula e/ou da cauda equina, que podem ser originadas por compressão direta por cisticercos e por reação inflamatória a distância, ou por degeneração da medula por paquimeningite ou por incapacidade circulatória. ${ }^{5}$ A maioria dos casos relatados de cisticercose intramedular atinge indivíduos entre 20 e 45 anos de idade; e a duração dos sintomas varia de uma semana a 10 anos. ${ }^{12,19}$ Nosso paciente tinha sintomatologia que se arrastava por mais de seis anos e tinha 42 anos. Sintomas comuns incluem: dor (nesse ponto, nosso paciente tinha uma particularidade especial: a anestesia), para ou quadriparesia, espasticidade, incontinência fecal e urinária e disfunção sexual. ${ }^{9}$ Eles podem ser acompanhados por uma variedade de déficits sensoriais. ${ }^{11,24}$ Esses sintomas podem ser secundários a efeito de massa das lesões, e sua ocorrência não deve ser atribuída principalmente à cisticercose intramedular. Esta pode ser a causa do diagnóstico tardio, principalmente em áreas não endêmicas, a menos que haja evidência de infecção concomitante por cisticercos noutro local. O diagnóstico diferencial de uma lesão cística intramedular em um paciente que se apresenta com dor nas costas é extensa e inclui neoplasias, ${ }^{7,8,11,19,25}$ doenças infecciosas (por exemplo, abscesso) ${ }^{16}$ e inflamatórias (por exemplo, esclerose múltipla), alterações medulares (por exemplo, cavita- ção siringomiélica) ${ }^{11,19} \mathrm{e}$ infestações parasitárias (por exemplo, cisticercose e cistos hidáticos). ${ }^{11}$

Para fazer o diagnóstico, os exames de imagens são primordiais. A RNM possui a vantagem de não empregar radiação ionizante, consente boa visualização de cistos intraventriculares, no tronco, cisterna pré-pontina, leptomeninges, lesão na base do cérebro e lesões intramedulares espinhais. No entanto, é inferior à tomografia axial computadorizada, na verificação de calcificações. Os achados da RNM expõem, na fase inicial, sinal de baixa intensidade ou da mesma intensidade do liquor cefalorraquidiano (LCR) para a maioria dos cistos intraventriculares e parenquimatosos viáveis. A fase inflamatória com edema e degeneração é representada pelo sinal de alta intensidade circunjacente às lesões. ${ }^{2}$

O melhor tratamento para a cisticercose intramedular continua controverso ${ }^{24}$. A excisão cirúrgica pode dar o diagnóstico definitivo, como pudemos comprovar, pois antes da cirurgia não tínhamos diagnóstico definitivo, e aliviar os sintomas compressivos ${ }^{2,5,6}$ é recomendado pela maioria dos autores como tratamento de escolha. ${ }^{4-6,12,19-21,25}$ Como os cistos geralmente têm localização superficial ( $3 \mathrm{~mm}$ de profundidade, ou menos), ${ }^{19}$ a ressecção total é possível na maioria dos casos. ${ }^{2,4}$ Alguns autores afirmam que o tratamento pós-operatório com fármacos anticisticercoides, como o albendazol, seria justificado, uma vez que cisticercose é uma doença generalizada, com sintomas focais. ${ }^{1,13,19,22}$

\section{Conclusão}

Portanto, deve-se ter em mente que, apesar da raridade, a possibilidade diagnóstica de neurocisticercose intramedular necessita ser aventada, pois muitas vezes o diagnóstico correto só é firmado após a exérese cirúrgica, tal qual nosso caso. A RNM é o exame de escolha, sempre lembrando que, apesar da sensibilidade, não se pode descartar a possibilidade de outras nosologias. O tratamento de eleição é o cirúrgico, apesar de, como vimos no caso de nosso paciente, nem sempre resultar na regressão dos sintomas.

\section{Referências}

1. Alsina GA, Johnson JP, McBride DQ, Rhoten PRL, Mehringer CM, Stokes JK. Spinal neurocysticercosis. Neurosurg Focus. 2002;12:1-7.

2. Andrade Filho AS, Souza APQU, Souza YMA. Neurocisticercose: diagnóstico. Revisão. Parte II. RBNP. 1998;2:9-12. 
3. Arruda WO, Ramina R, Pedrozo AA, Meneses MS. Brainstem cysticercosis simulating cystic tumor lesion. Arq Neuropsiquiatr. 1994;52:431-4.

4. Barini O. Cisticerco macrocístico intramedular: extirpação cirúrgica. Arq Neuropsiquiatr. 1954;12:264-6.

5. Colli BO, Assirati Jr JA, Machado HR, Santos F, Takayanagui OM. Cysticercosis of the central nervous system: II. Spinal cysticercosis. Arq Neuropsiquiatr. 1994;52:187-99.

6. Colli BO, Valença MM, Carlotti Jr CG, Machado HR, Assirati Jr JA. Spinal cord cysticercosis: neurosurgical aspects. Neurosurg Focus. 2002;12:1-7.

7. Corral I, Quereda C, Moreno A. Intramedullary cysticercosis cured with drug treatment. Spine. 1996;19:2284-7.

8. Dantas FLR, Fagundes-Pereyra WJ, Souza CT, Vega MG, Souza AA. Cisticercose intramedular: relato de caso. Arq Neuropsiquiatr. 1999;57:301-5.

9. Gallani NR, Zambelli HJL, Roth-Vargas AA, Limoli C Jr. Cysticercosis of the spinal cord: report of two cases, literature review and comments on pathogenesis. Arq Neuropsiquiatr. 1992;50:343-50.

10. Holanda LF. Infecções do sistema nervoso central. In: Holanda LF, editor. Manual de neurocirurgia. São Paulo: Editora BYK; 1995, p. 13-159.

11. Leite CC, Jinkins JR, Escobar BE. MR imaging of intramedullary and intradural-extramedullary spinal cysticercosis. Am J Roentgenol. 1997;169:1713-7.

12. Mathuriya SN, Khosla VK, Vasishta RK, Tewari MK, Pathak A, Prabhakar S. Intramedullary cysticercosis: MRI diagnosis. Neurol India. 2001;49:71-4.

13. Mohanty A, Venkatrama SK, Das S, Das BS, Rao BR, Vasudev MK. Spinal intramedullary cysticercosis. Neurosurgery. 1997;1:82-87.

14. Pittella JE. Neurocysticercosis. Brain Pathol. 1997;7:681-93.

15. Queiroz LS, Pellegrinni Filho A, Callegaro D, Faria LL. Intramedullary cysticercosis. J Neurol Sci. 1975;26:61-70.
16. Robertson HJ, Watson J. Case 4: neurocysticercosis with cervical meningeal involvement. Am J Roentgenol. 1998;171:879-80.

17. Rossitti SL, Roth-Vargas AA, Moreira ARS, Sperlescu A, Araújo JFM, Balbo RJ. Cisticercose espinhal leptomeníngea pura. Arq Neuropsiquiatr. 1990;48:366-70

18. Sharma BS, Banerjee AK, Kak VK. Intramedullary spinal cysticercosis: case report and review of literature. Clin Neurol Neurosurg. 1987;89:111-6.

19. Sheehan JP, Sheehan J, Lopes MB, Jane JA. Intramedullary spinal cysticercosis: case report and review of the literature. Neurosurg Focus. 2002;12:1-4.

20. Siqueira MC, Koury LS, Boer CAA, Rezende-Filho CP. Cisticerco solitário intramedular: relato de caso e revisão da literatura. Arq Bras Neurocir (São Paulo). 1987;8:131-9.

21. Singh NN, Verma R, Pankaj BK, Misra S. Cauda-conus syndrome resulting from neurocysticercosis. Neurol India. 2003;51:118-20.

22. Singh P, Sahai K. Intramedullary cysticercosis. Neurol India. 2004;52:264-5.

23. Takayanagui OM. Neurocisticercose: evolução clínico-laboratorial de 151 casos [tese]. Ribeirão Preto, SP. Faculdade de Medicina de Ribeirão Preto - Universidade de São Paulo; 1987.

24. Torabi AM, Quiceno M, Mendelsohn DB, Powell CM Multilevel intramedullary spinal neurocysticercosis with eosinophilic meningitis. Arch Neurol. 2004;61:770-2.

25. Vázquez MAS, Carachure IJ, Maltos URC, Herrera FS Cisticercosis intramedular. Arch Bras Neurocir. 2001;16:36-8.

Endereço para correspondência

Luciano Ferreira de Holanda

Rua Delmiro Gouveia, 299

58107-735 - Campina Grande, PB, Brasil

E-mail:luholanda@hat.com.bre benedito.jamilson@hotmail.com

Telefone: (+55 83) 9149-6767 